\title{
Prevalence and risk factors of superior segmental optic hypoplasia in a Korean population: the Korea National Health and Nutrition Examination
}

\section{Survey}

Sam Seo ${ }^{1}$, Chong Eun Lee ${ }^{1}$, Dai Woo Kim¹, Young Kook Kim², Jin Wook Jeoung ${ }^{1}$, Chan Yun Kim³, Se Woong Kang ${ }^{4}$, Ki Ho Park ${ }^{1 *}$ and Epidemiologic Survey Committee of the Korean Ophthalmological Society

\begin{abstract}
Background: This study investigated the prevalence and risk factors for superior segmental optic hypoplasia (SSOH) in a Korean population based on the data from the nationwide Korea National Health and Nutrition Examination Survey (KNHANES).

Methods: We performed a retrospective review of the KNHANES dataset covering January 2012 to December 2012. The study population comprised 5,612 subjects ( $\geq 19$ years of age) who had participated in a medical interview covering demographic and systemic information, been issued a questionnaire regarding associated $\mathrm{SSOH}$ risk factors including gender, age, systemic disease, and family history, and had undergone an ophthalmologic examination. Two masked readers evaluated fundus photography, paying special attention to the presence of $\mathrm{SSOH}$. Associations of risk factors (identified in the medical interview portion) with $\mathrm{SSOH}$ prevalence were investigated using multivariate logistic regression analysis.

Results: $\mathrm{SSOH}$ was detected in 16 eyes of 14 subjects, or $0.24 \%$ of the 5,612 subjects. All 16 eyes showed a corresponding visual-field defect. In multivariate logistic regression analyses, maternal history of diabetes (Odds ratio (OR), 7.666; 95\% Confidence interval $(\mathrm{Cl}), 2.601 \sim 22.593, \mathrm{p}<0.001)$ and paternal history of ischemic heart disease (IHD) (OR, 11.105; $\mathrm{Cl}$, $3.361 \sim 36.686, p<0.001$ ) were associated with increased risk of SSOH.

Conclusions: This study provides the first representative population-based data on $\mathrm{SSOH}$ prevalence in Korea. Additionally, multivariate analyses revealed that a history of maternal diabetes and paternal IHD was the most important factor influencing the prevalence of $\mathrm{SSOH}$.
\end{abstract}

Keywords: Superior segmental optic hypoplasia, Prevalence, Risk factor, Korea National Health and Nutrition Examination survey

\section{Background}

Superior segmental optic hypoplasia ( $\mathrm{SSOH}$ ) is a nonprogressive congenital optic nerve anomaly characterized by a relative superior entrance of the central retinal artery, pallor of the superior optic disc, superior peripapillary scleral halo, and thinning of the superior retinal nerve

\footnotetext{
* Correspondence: kihopark@snu.ac.kr

'Department of Ophthalmology, Seoul National University Hospital, Seoul National University College of Medicine, 101 Daehak-roJongno-gu, Seoul 110-744, Korea

Full list of author information is available at the end of the article
}

fiber layer (RNFL) [1]. Patients with SSOH typically have good visual acuity and an inferiorly located visual-field defect in the affected eyes [2].

Petersen \& Walton described a specific form of optic nerve hypoplasia characterized by a relative hypoplasia of the superior portion of the optic nerve head [3]. The term superior segmental optic hypoplasia, or $\mathrm{SSOH}$, became generally accepted after Kim et al.'s investigation of this condition's association with maternal type I diabetes mellitus [1]. The pathogenesis of this condition, however, remains unclear. Although association with maternal diabetes 
has been strongly suggested, cases occurring in the absence of maternal diabetes also been reported [2,3].

Yamamoto et al. recently reported a Japanese $\mathrm{SSOH}$ prevalence rate of $0.3 \%$ [4]. On the basis of this relatively high prevalence, they emphasized the need for greater awareness in clinical practice. In this light, we considered it prudent to undertake a study on the prevalence and risk factors for SSOH in Korea.

In the present study, we determined the prevalence of $\mathrm{SSOH}$ using data from the Korean National Health and Nutrition Examination Survey (KNHANES), a large population-based, cross-sectional survey. Additionally, we investigated the ocular and systemic parameters to identify the risk factors associated with $\mathrm{SSOH}$.

\section{Methods}

\section{Sample and population}

The data used in the present study were obtained from KNHANES 2012, which covers the months from January to December of that year. The KNHANES a cross-sectional and nationally representative survey that was conducted by the Ministry of Health and Welfare of the Republic of Korea and the Korean Ophthalmological Society. Using a multistage, stratified, probability-clustered sampling method and a weighting scheme, the detailed survey design can produce estimated health statistics representative of the civilian, noninstitutionalized Korean population. This study has followed the Tenets of the Declaration of Helsinki. As the data of KNHANES is opened to the public after removal of personal identifiers and being anonymized (http://knhanes.cdc. go.kr), the Institutional Review Board of Seoul National University Hospital determined that this study was exempt from requiring their approval. This study included KNHANES data on 5,612 individuals aged 19 years or older.

\section{Survey components}

All of the participants underwent a personal medical interview and an ophthalmological examination as part of the KNHANES process. The interview covered demographic and systemic information. For assessment of overall general medical condition, including any history of ocular disease, systemic disease, family history and current medication, a standardized questionnaire was administered.

Participants were asked whether any biological member of their family, living or decreased, had ever been told he/ she had diabetes, hypertension, or ischemic heart disease (IHD). We defined family history as having a first-degree relative (parent) with diabetes, hypertension, or IHD diagnosed by a doctor and treated with medication, regardless of disease severity or duration. Family history was categorized as maternal and paternal.

The full ophthalmologic examination included logMAR visual acuity measurements, auto-refraction, slit-lamp examination for anterior segment of the eye, intraocular pressure (IOP) measurements, and fundus photography. Refraction measurements were converted into spherical equivalents and calculated as the spherical value plus half the astigmatic value. IOP was measured with a Goldmann applanation tonometer (Haag-Streit, Inc., Bern, Switzerland). Digital fundus images were taken with a digital nonmydriatic fundus camera (TRC-NW6S; Topcon, Inc., Tokyo, Japan).

Visual-field testing was performed with frequencydoubling technology (FDT) (Humphrey Matrix; Carl Zeiss Meditec, Inc., Dublin, CA) using the N-30-1 screening test. The test location was deemed abnormal if not identified on two attempts at a contrast level identified by $99 \%$ of the healthy population.

Two independent readers who were masked to all information other than the fundus photographs, evaluated the color fundus photography for the presence of abnormalities of the optic nerve head and RNFL. Discrepancies between the two observers' findings were resolved by consensus. In case of no consensus the final decision was made by the third reader, the principal investigator. In this study, $\mathrm{SSOH}$ was defined as rim thinning of the optic nerve head in the superior or superonasal region with corresponding RNFL defects in at least one eye [4].

\section{Statistical analysis}

Fisher's exact test was utilized to compare the demographic characteristics. A multivariate logistic regression models were applied to the determination of the $\mathrm{SSOH}$ risk factors, adjusting for potential confounders. Adjusted means, 95\% CIs, and 2 sided P values were obtained. Statistical significance was defined as a $\mathrm{P}$ value less than or equal to 0.05 .

\section{Results}

During the 12-month KNHANES period, 5,612 participants aged 19 years or older underwent fundus photography. In the present study, fundus photographs of 11,087 eyes of those 5,612 cases were successfully reviewed. 137 eyes was excluded due to media opacities or other difficulties such as small pupil or poor focus. The age and gender distributions of the study participants are shown in Table 1. Optic disc anomalies were detected in 53 eyes of 48 subjects with a prevalence rate of $0.48 \%$ per eye and $0.86 \%$ per subject. Optic disc drusen were detected on the fundus photographs of 10 eyes of nine patients. Myelinated nerve fibers were detected in 27 eyes of 24 subjects. Of the 5,612 cases reviewed, we found 16 eyes of 14 cases with $\mathrm{SSOH}$ (about $0.14 \%$ of the eyes, and about $0.24 \%$ of the cases). All of the 16 eyes showed corresponding inferior visual-field defect. SSOH affected both eyes in two cases and one eye in 12 cases. The clinical features of the $\mathrm{SSOH}$ participants are provided in Table 2. The visual acuity in the $\mathrm{SSOH}$ group was $0.07 \pm 0.10$, and in the 
Table 1 Participants by age and gender

\begin{tabular}{llll}
\hline Age (years) & Men & Women & Total \\
\hline $19-29$ & 258 & 381 & 639 \\
$30-39$ & 383 & 555 & 938 \\
$40-49$ & 397 & 550 & 947 \\
$50-59$ & 444 & 632 & 1076 \\
$60-69$ & 433 & 580 & 1013 \\
$70-79$ & 362 & 469 & 831 \\
$\geq 80$ & 57 & 111 & 168 \\
Total & 2334 & 3278 & 5612 \\
\hline
\end{tabular}

normal group, $0.17 \pm 0.33$. The worst visual acuity in the $\mathrm{SSOH}$ group was 0.3. The SSOH group showed lower logMAR scores (better visual acuity) than were recorded for the normal group. This finding is consistent with a previous study, which showed that patients with $\mathrm{SSOH}$ typically have good visual acuity. The mean refraction in the $\mathrm{SSOH}$ group was $-1.06 \pm 1.86 \mathrm{D}$, which was similar to that in the normal group $(-0.80 \pm 2.17 \mathrm{D})$.

The results of the ocular and systemic parameter comparison are summarized in Table 3. The participants' ages ranged from 19 to 65 years (mean \pm SD: $43.50 \pm 14.89$ years). The visual acuity was better than or equal to $20 / 20$ in eight $(57.14 \%)$ and worse than $20 / 25$ in three (21.43\%) of the 14 eyes with $\mathrm{SSOH}$. When the randomized one eye was selected in bilateral cases and the affected eyes were selected in unilateral cases, the refractive error as spherical equivalent was $-1.06 \pm 1.86 \mathrm{D}$ (mean $\pm \mathrm{SD}$; range: -4.38 to 2.25 $\mathrm{D}$ ); the IOP was $14.68 \pm 2.60 \mathrm{mmHg}$ (mean $\pm \mathrm{SD}$; range: 10 to $19 \mathrm{mmHg}$ ). There was no statistically significant difference between $\mathrm{SSOH}$ and the controls in age, gender, history of hypertension, diabetes or IHD.

A Fisher's exact test revealed that a paternal history of hypertension and IHD and a maternal history of diabetes were significant risk factors for $\mathrm{SSOH}$ (Table 4). In the final logistic regression model for SSOH risk, the independent factors that remained significant were paternal history of IHD and maternal history of diabetes (Table 5). Paternal history of IHD (OR, 11.105; 95\% CI, $3.361 \sim 36.686)$ and maternal history of diabetes (OR, 7.666; 95\% CI, $2.601 \sim$ 22.593) were both associated with increased risk of $\mathrm{SSOH}$. Paternal history of hypertension did not significantly affect predisposition to $\mathrm{SSOH}$.

\section{Discussion}

This study analyzed the data from a national health survey (KNHANES) and determined the prevalence and risk factors for $\mathrm{SSOH}$. SSOH prevalences have been reported in a number of Asian studies. Yamamoto et al. reported that prevalence of $\mathrm{SSOH}$ in Japan, as determined by fundus photography, was $0.3 \%$ among their subjects and $0.2 \%$ among the general population [4]. Han et al., reviewing photographs of 3,905 hospital-based Korean subjects, calculated a $0.08 \%$ prevalence [5]. However, those study subjects were not representative of the general Korean population but only of the one institute where they were examined.

Our study demonstrated that the prevalence of $\mathrm{SSOH}$ in Korea is actually as high as $0.24 \%$. The discrepancy in the prevalence of $\mathrm{SSOH}$ between the present study and

Table 2 Clinical data on superior segmental optic hypoplasia (SSOH) cases reviewed in present study

\begin{tabular}{llllll}
\hline No. & Age (years) & Laterality & BCVA (logMAR) & IOP & Refraction (SE; Diopters) \\
\hline 1 & 19 & OS & 0 & 15 & -3.75 \\
2 & 24 & OD & 0 & 15 & -1.50 \\
$3-1$ & 29 & OD & 0.1 & 16 & 2.25 \\
$3-2$ & 29 & OS & 0.1 & 16 & 1.75 \\
4 & 34 & OD & 0.2 & 15 & -3.25 \\
5 & 38 & OD & 0 & 14 & -3.75 \\
6 & 38 & OD & 0 & 18 & -1.25 \\
7 & 41 & OD & 0.1 & 13 & -0.25 \\
$8-1$ & 42 & OD & 0 & 13 & -0.50 \\
$8-2$ & 42 & OS & 0 & 10 & -0.63 \\
9 & 44 & OS & 0 & 11 & -1.50 \\
10 & 49 & OS & 0 & 16 & 2.25 \\
11 & 59 & OS & 0 & 19 & -0.56 \\
12 & 63 & OD & 0.2 & 17 & -4.38 \\
13 & 64 & OD & 0.1 & 0.3 & -2.63 \\
\hline
\end{tabular}

$\mathrm{BCVA}=$ Best corrected visual acuity; logMAR = Logarithm of the Minimum Angle of Resolution; SE = Spherical equivalent; IOP = Intraocular pressure. 
Table 3 Parameter differences between SSOH and healthy subjects

\begin{tabular}{llll}
\hline & SSOH subjects $(\mathbf{n}=\mathbf{1 4}$ Subjects) & Healthy subjects ( $\mathbf{n = 5 , 5 9 8 ~ S u b j e c t s )}$ & $\boldsymbol{P}_{\text {value }}{ }^{*}$ \\
\hline Ocular & & & \\
$\quad$ Refractive error (SE, diopter) & $-1.06 \pm 1.86$ & $-0.80 \pm 2.17$ & 0.261 \\
$\quad$ Intraocular pressure (mmHg) & $14.68 \pm 2.60$ & $13.78 \pm 2.68$ & 0.192 \\
Systemic & & & 0.079 \\
Age (years) & $43.50 \pm 14.89$ & $51.55 \pm 16.81$ & 0.923 \\
Gender (female) & $8 / 14$ & $3270 / 5598$ & 0.379 \\
Systemic hypertension & $2 / 14$ & $1366 / 5598$ & 0.446 \\
Ischemic heart disease & $1 / 14$ & $192 / 5598$ & 0.248 \\
Diabetes & $0 / 14$ & $488 / 5598$ & \\
\hline
\end{tabular}

$\mathrm{SSOH}=$ superior segmental optic hypoplasia; $\mathrm{SE}=$ Spherical equivalent.

The numerical values are shown as the mean \pm standard deviation (with the range in parentheses).

The values of refractive error and intraocular pressure (IOP) were randomly selected for both eyes.

${ }^{*} \mathrm{P}$ value based on Mann-Whitney $\mathrm{U}$ test, $\mathrm{P}<0.05$ significant.

Han's study is attributable to the difference in the study population. Our study participants constituted a representative Korean population, while those in Han's study are patients who had visited a health promotion center in a tertiary referral hospital. Han et al. noted, as a limitation, the possibility that participants with a known optic disc anomaly might not have been included. Additionally, different examinations were used for $\mathrm{SSOH}$ diagnosis. A combination of stereo disc photography, red-free retinal nerve fiber layer, and Humphrey visual field test was used in the study by Han et al., whereas fundus photography and frequency-doubling technology (FDT) perimetry were used in our study. The difference in the diagnostic ability between these examination sets would have led to the difference in the prevalence.

In fact, ours is the first study to investigate the Korean prevalence of $\mathrm{SSOH}$ in a representative random population. Considering the ethnic similarity between Koreans and Japanese along with Yamamoto et al.'s result, we believe that the prevalence of $\mathrm{SSOH}$ obtained in our study is an accurate estimate of its true prevalence.

$\mathrm{SSOH}$ has been noted to occur both unilaterally and bilaterally with relatively equal frequency $[2,6]$. The percentage of unilateral $\mathrm{SSOH}$ in our series was slightly higher than those reported by other investigators. There were no statistically significant differences in prevalence by age or gender. Although several reports have indicated a female predisposition to SSOH $[1,3,7]$, no significant gender difference has yet been documented anywhere. Further research will be necessary for helpful clarification of such issues.

Tendencies toward lower birth weight, shorter gestation time and poorer control of maternal diabetes were associated with increased risk of $\mathrm{SSOH}$ [8]. These associations could be due to unfavorable conditions during development that affect fetal growth, general fetal development

Table 4 Associations between SSOH prevalence and systemic factors by cross-tables

\begin{tabular}{|c|c|c|c|c|}
\hline Factor & SSOH subjects ( $n=14$ Subjects) & Healthy subjects ( $n=5,598$ Subjects) & Odds ratio $(95 \% \mathrm{Cl})$ & P-value ${ }^{*}$ \\
\hline \multicolumn{5}{|l|}{ Systemic } \\
\hline HTN & $2 / 14$ & $1366 / 5598$ & $0.516(0.115-2.310)$ & 0.539 \\
\hline $\mathrm{HD}$ & $1 / 14$ & $192 / 5598$ & $2.166(0.282-16.641)$ & 0.388 \\
\hline Diabetes & $0 / 14$ & $488 / 5598$ & & 0.627 \\
\hline \multicolumn{5}{|l|}{ Family history } \\
\hline Paternal HTN & $5 / 14$ & $853 / 5598$ & $3.090(1.033-9.244)$ & 0.05 \\
\hline Maternal HTN & $4 / 14$ & $1113 / 5598$ & $1.612(0.505-5.149)$ & 0.498 \\
\hline Paternal IHD & $4 / 14$ & $155 / 5598$ & $14.046(4.358-45.279)$ & $<0.001$ \\
\hline Maternal IHD & $1 / 14$ & $150 / 5598$ & $2.794(0.363-21.495)$ & 0.318 \\
\hline Paternal diabetes & $1 / 14$ & $450 / 5598$ & $0.880(0.115-6.742)$ & 1.0 \\
\hline Maternal diabetes & $6 / 14$ & $428 / 5598$ & $9.060(3.129-26.230)$ & $<0.001$ \\
\hline
\end{tabular}

$\mathrm{SSOH}=$ superior segmental optic hypoplasia; $95 \% \mathrm{Cl}=95 \%$ confidence interval. 
Table 5 Logistic regression analysis of $\mathrm{SSOH}$-associated factors

\begin{tabular}{llll}
\hline Factor & Odds ratio & $\mathbf{9 5 \%} \mathbf{C l}$ & $\boldsymbol{P}$ value $^{*}$ \\
\hline Paternal history of HTN & 2.249 & $0.726-6.961$ & 0.160 \\
Paternal history of IHD & 11.105 & $3.361-36.686$ & $<\mathbf{0 . 0 0 1}$ \\
Maternal history of diabetes & 7.666 & $2.601-22.593$ & $<\mathbf{0 . 0 0 1}$
\end{tabular}

$\mathrm{SSOH}=$ superior segmental optic hypoplasia; $95 \% \mathrm{Cl}=95 \%$ confidence interval. $\mathrm{HTN}=$ systemic hypertension; IHD = ischemic heart disease.

*P-values based on logistic regression; $\mathrm{P}<0.05$ significant.

Significant values are shown in bold.

and the risk of malformation. Brodsky et al. suggested, based on cases of identical twins, genetic susceptibility as a possible risk of SSOH [6]. Unoki et al. also noted a familial case of $\mathrm{SSOH}$ [9]. In any case, the pathogenic mechanism of SSOH remains obscure.

The results of this study indicated that maternal history of diabetes and paternal history of IHD are significantly associated with $\mathrm{SSOH}$. Whereas reported risk factors for $\mathrm{SSOH}$ have been relatively rare in the literature, several studies have shown a correlation between insulin-dependent diabetic mothers and $\mathrm{SSOH}$ in their children $[7,8]$. Our present study served to reconfirm the positive relationship between maternal history of diabetes and SSOH in children. A possible pathology is selective interference of the teratologic mechanism of insulin-dependent diabetes mellitus with the early-gestational development of superior retinal ganglion cells.

As for paternal history of IHD, it has gone completely unreported in the literature, and remains to be confirmed in future studies. Our present results, uniquely, showed paternal history of IHD to be an independent risk factor for $\mathrm{SSOH}$. A possible explanation is related to altered levels of growth factors and insulin sensitivity. Several studies have reported an association between optic nerve hypoplasia and endocrine disturbances including growth hormone $(\mathrm{GH})$ deficiency and insulin resistance $[10,11]$. Paternal IHD has been reported for offspring with insulin resistance [12], which association could increase the risk of optic nerve hypoplasia.

Paternal IHD was found to be associated with the GH level of offspring. Previously, paternal IHD has been reported for offspring with polymorphism in angiotensin converting enzyme (ACE) [13]. ACE variation, moreover, has been associated with lower levels of growth hormone and insulin-like growth factors, because the ACE polymorphism site is located nearby the GH gene site [14]. It is not clear if the finding of the association with the $\mathrm{GH}$ level of offspring, then, would explain an increased risk of SSOH. However, this association is a possible explanation of genetic susceptibility to SSOH. Because determinative genetic information could not be obtained in this study, and because the association between paternal IHD and congenital malformation remains controversial, further study in efforts to prove that association certainly is warranted.

This study has several limitations. The analysis relied on self-reported data on medical conditions. As a result, the prevalences could have been underestimated or influenced by the individual's memory. Another limitation of the current analysis is that we were unable to assess disease onset, duration, or severity, as pertinent data were not collected by KNHANES.

Notwithstanding the limitations of the present study, the strength of this study is that the sample size is significantly large, and indeed, KNHANES is based on a representative sample of the Korean population. Therefore, the proportions and odds ratios obtained in this study are representative of the Korean population as well.

\section{Conclusions}

We determined that the prevalence of SSOH in Korea is $0.24 \%$. To our knowledge, this is the first study on $\mathrm{SSOH}$ prevalence based on data representing a Korean population (KNHANES). We conclude that individuals with a history of maternal diabetes and paternal IHD might be at a higher risk of $\mathrm{SSOH}$.

\section{Abbreviations \\ SSOH: Superior segmental optic hypoplasia; KNHANES: Korea National Health and Nutrition Examination Survey; OR: Odds ratio; Cl: Confidence interval; IHD: Ischemic heart disease; RNFL: Retinal nerve fiber layer; IOP: Intraocular pressure; FDT: Frequency doubling technology.}

\section{Competing interests}

The authors declare that they have no competing interests.

\section{Authors' contributions}

SS conceived of the study, and participated in its design and coordination, and helped to draft the manuscript. CEL participated in its design and coordination and performed the statistical analysis, and helped to draft the manuscript. YKK and DWK made substantial contributions to analysis and interpretation of data. CYK and SWK participated in the design of the study and performed the statistical analysis. JWJ and KHP participated in the design of the study and give final approval of the version to be published. All authors read and approved the final manuscript.

\section{Acknowledgments}

We are grateful to the patients for their participation in this research.

\section{Author details}

${ }^{1}$ Department of Ophthalmology, Seoul National University Hospital, Seoul National University College of Medicine, 101 Daehak-roJongno-gu, Seoul 110-744, Korea. ${ }^{2}$ Department of Ophthalmology, Jeju National University Hospital, 15, Aran-13-gil, Jeju-si, Jeju 690-767, Korea. ${ }^{3}$ The Institute of Vision Research, Department of Ophthalmology, Yonsei University College of Medicine, 50 Yonsei-ro, Seodaemun-gu, Seoul, Korea. ${ }^{4}$ Department of Ophthalmology, Samsung Medical Center, Sungkyunkwan University School of Medicine, 81 Irwon-ro, Gangnam-gu, Seoul, Korea.

Received: 13 May 2014 Accepted: 11 December 2014

Published: 15 December 2014

\section{References}

1. Kim RY, Hoyt WF, Lessell S, Narahara MH: Superior segmental optic hypoplasia. A sign of maternal diabetes. Arch Ophthalmol 1989, 107:1312-1315. 
2. Hashimoto M, Ohtsuka K, Nakagawa T, Hoyt WF: Topless optic disk syndrome without maternal diabetes mellitus. Am J Ophthalmol 1999, 128:111-112.

3. Petersen RA, Walton DS: Optic nerve hypoplasia with good visual acuity and visual field defects: a study of children of diabetic mothers. Arch Ophthalmol 1977, 95:254-258.

4. Yamamoto T, Sato M, Iwase A: Superior segmental optic hypoplasia found in Tajimi Eye Health Care Project participants. Jpn J Ophthalmol 2004, 48:578-583.

5. Han SB, Park KH, Kim DM, Kim TW: Prevalence of superior segmental optic nerve hypoplasia in Korea. Jpn J Ophthalmol 2009, 53:225-228.

6. Brodsky MC, Schroeder GT, Ford R: Superior segmental optic hypoplasia in identical twins. J Clin Neuroophthalmol 1993, 13:152-154.

7. Nelson M, Lessell S, Sadun AA: Optic nerve hypoplasia and maternal diabetes mellitus. Arch Neurol 1986, 43:20-25.

8. Landau K, Bajka JD, Kirchschlager BM: Topless optic disks in children of mothers with type I diabetes mellitus. Am J Ophthalmol 1998, 125:605-611.

9. Unoki K, Ohba N, Hoyt WF: Optical coherence tomography of superior segmental optic hypoplasia. Br J Ophthalmol 2002, 86:910-914.

10. Skarf B, Hoyt CS: Optic nerve hypoplasia in children. Association with anomalies of the endocrine and CNS. Arch Ophthalmol 1984, 102:62-67.

11. Costin G, Murphree AL: Hypothalamic-pituitary function in children with optic nerve hypoplasia. Am J Dis Child 1985, 139:249-254

12. Khalil A, Prakash $V$, Bhattacharjee J: Insulin resistance and lipid profile in the children of young ischemic parents. Indian Pediatr 2003, 40:946-950

13. Tiret L, Kee F, Poirier O, Nicaud V, Lecerf L, Evans A, Cambou JP, Arveiler D, Luc G, Amouyel P, Cambien F: Deletion polymorphism in angiotensinconverting enzyme gene associated with parental history of myocardial infarction. Lancet 1993, 341:991-992.

14. Puthucheary Z, Skipworth JR, Rawal J, Loosemore M, Van Someren K Montgomery HE: The ACE gene and human performance: 12 years on. Sports Med 2011, 41:433-448.

doi:10.1186/1471-2415-14-157

Cite this article as: Seo et al:: Prevalence and risk factors of superior segmental optic hypoplasia in a Korean population: the Korea National Health and Nutrition Examination Survey. BMC Ophthalmology 2014 14:157.

\section{Submit your next manuscript to BioMed Central and take full advantage of:}

- Convenient online submission

- Thorough peer review

- No space constraints or color figure charges

- Immediate publication on acceptance

- Inclusion in PubMed, CAS, Scopus and Google Scholar

- Research which is freely available for redistribution 